\title{
SIRT5 as a biomarker for response to anthracycline-taxane-based neoadjuvant chemotherapy in triple-negative breast cancer
}

\author{
LU XU ${ }^{1,2}$, XIAOFANG CHE ${ }^{1,2}$, YING WU $^{3}$, NA SONG ${ }^{1,2}$, SHA SHI $^{1,2}$, SHUO WANG $^{1,2}, \mathrm{CE} \mathrm{LI}^{1,2}$, \\ LINGYUN ZHANG ${ }^{1,2}$, XINLIAN ZHANG ${ }^{4}$, XIUJUAN QU ${ }^{1,2}$ and YUEE TENG ${ }^{1,2}$ \\ ${ }^{1}$ Department of Medical Oncology, ${ }^{2}$ Key Laboratory of Anticancer Drugs and Biotherapy of \\ Liaoning Province, ${ }^{3}$ Department of General Practice, The First Hospital of China Medical University, Shenyang, \\ Liaoning 110001, P.R. China; ${ }^{4}$ Department of Statistics, University of Georgia, Athens, GA 30602, USA
}

Received September 18, 2017; Accepted February 28, 2018

DOI: $10.3892 /$ or.2018.6319

\begin{abstract}
Neoadjuvant chemotherapy (NAC) is of great importance for patients with triple-negative breast cancer (TNBC) and the achievement of pathological complete response (pCR) to NAC in TNBC patients indicates survival benefits. However, the identification of reliable predictive biomarkers of pCR to NAC in TNBC patients remains an urgent and largely unattended medical issue. In the present study, we evaluated the differentially expressed genes (DEGs) between pCR and non-pCR patients after doxorubicin/cyclophosphamide therapy, followed by paclitaxel pre-operative treatment in 64 TNBC patients recorded in the GSE41998 dataset of Gene Expression Omnibus and identified 118 DEGs. Subsequently, we selected five core genes that were closely associated with the pCR of TNBC patients by using a genetic algorithm-support vector machine-based method. Sirtuin 5 (SIRT5) was one of the five core genes and patients who achieved pCR expressed higher levels of SIRT5. Thus, we speculated that SIRT5 may be a potential predictive marker of the response to anthracycline-taxane-based chemotherapy. Oncomine analysis revealed that the expression levels of SIRT5 were higher in epirubicin/cyclophosphamide-docetaxel
\end{abstract}

Correspondence to: Professor Yuee Teng, Department of Medical Oncology, The First Hospital of China Medical University, 155 North Nanjing Street, Heping, Shenyang, Liaoning 110001, P.R. China

E-mail: yeteng@cmu.edu.cn

Abbreviations: SIRT5, sirtuin 5; NAC, neoadjuvant chemotherapy; TNBC, triple-negative breast cancer; ER, estrogen receptor; PR, progesterone receptor; HER2, human epidermal growth factor receptor 2; pCR, pathological complete response; DEGs, differentially expressed genes; GA-SVM, genetic algorithm-support vector machine; RFS, relapse-free survival; PTMs, post-translational modifications

Key words: triple-negative breast cancer, neoadjuvant chemotherapy, pathological complete response, genetic algorithm-support vector machine classifier, sirtuin 5, Oncomine database responders compared with non-responders. Furthermore, Gene Ontology analysis indicated that SIRT5 may affect the response to anthracycline-taxane-based chemotherapy by regulating the Rho pathway. It was also observed that SIRT5 was upregulated in TNBC and breast cancer with BRCA1 mutation subtypes. High SIRT5 expression was also associated with poor clinical outcomes of breast cancer patients. In conclusion, the present study revealed SIRT5 as a biomarker for response to anthracycline-taxane-based NAC in patients with TNBC and identified a series of novel biological functions of SIRT5 in breast cancer.

\section{Introduction}

Triple-negative breast cancer (TNBC) accounts for $\sim 15 \%$ of all breast cancers and is characterized by the absence of estrogen receptor (ER), progesterone receptor (PR) and human epidermal growth factor receptor 2 (HER2) expression (1). Without effective therapeutic targets, chemotherapy is the only systemic treatment strategy for patients with TNBC. Neoadjuvant chemotherapy (NAC) for breast cancer is an important therapeutic modality that can improve the clinical outcome of individuals with breast cancer, particularly those with TNBC (2). It is widely accepted that pathological complete response (pCR) is a reliable predictor of good prognosis in patients with TNBC and a number of studies have investigated potential biomarkers for the pCR of TNBC patients to NAC (3-6). However, these investigations mainly focused on clinicopathological parameters, as well as imaging data and lymphocyte infiltration. Since its advent and development, high-throughput sequencing has been widely used to investigate the underlying mechanisms of numerous disorders. In particular, microarray analysis has dual significance, as it may be used to identify individuals with a higher chance of pCR to NAC and to explore potential therapeutic targets in TNBC patients, by using high-throughput sequencing data to identify reliable biomarkers.

At present, anthracycline-taxane-based NAC is among the most commonly administered regimens for patients with TNBC. Thus, there is an urgent necessity to identify reliable biomarkers for predicting the chemotherapeutic response and clinical outcome of TNBC patients following 
taxane-anthracycline-based NAC, in order to avoid the administration of toxic agents and to help doctors tailor treatment strategies for individual patients. Therefore, in the present study, we extracted gene expression data on 64 TNBC patients (with pCR and non-pCR to NAC) from the GSE41998 dataset of Gene Expression Omnibus (GEO), and identified five core genes that were closely associated with the pCR of TNBC patients, namely NOL7, GFER, COMMD4, sirtuin 5 (SIRT5) and SRC, via a genetic algorithm-support vector machine (GA-SVM)-based method.

SIRT5 is a member of the sirtuins (SIRTs), a family of nicotinamide adenine dinucleotide $\left(\mathrm{NAD}^{+}\right)$-dependent deacetylases that participate in various biological processes, including the regulation of metabolism, cell division, aging and oxidative stress (7). In recent years, studies have evaluated the potential roles of SIRTs in different cancer types (8-10). In particular, the expression of SIRT5 has been assessed in various types of cancer, including endometrial carcinoma, head and neck squamous cell carcinoma, basal cell carcinoma, lung and breast cancer (11-14). However, to the best of our knowledge, no study has investigated the value of SIRT5 in predicting the pCR of TNBC patients to NAC, or the potential association of SIRT5 expression with the clinicopathological characteristics and prognosis of breast cancer patients.

In the present study, we aimed to clarify the relevance of SIRT5 expression to the pCR to NAC via online database analyses. Using bioinformatic methods, public microarray data were downloaded to determine whether the expression of SIRT5 was associated with the pCR to anthracycline-taxane-based NAC in TNBC patients. The biological functions of SIRT5 were also assessed by Gene Ontology (GO) analysis. Additionally, Oncomine database analysis was used to demonstrate whether the differential expression of SIRT5 in breast cancer patients was associated with the clinicopathological features, clinical outcomes and response to anthracycline-taxane-based chemotherapy in breast cancer patients.

\section{Materials and methods}

GEO dataset. The gene expression profile microarray dataset GSE41998, which was originated from a randomized, multicenter, open-label, phase II trial (NCT00455533), was obtained from the GEO database (www.ncbi.nlm.nih.gov/geo). This dataset was deposited by Horak et al (15), who enrolled previously untreated women with histologically-confirmed primary invasive breast adenocarcinoma and treated them with four cycles of AC (doxorubicin plus cyclophosphamide), followed by $1: 1$ randomization to ixabepilone $(n=148)$ or paclitaxel $(n=147)$. The gene expression data from 64 TNBC patients who received the AC-Taxol (paclitaxel) regimen were analyzed in this study. The dataset was originally produced using an Affymetrix Human Genome U133A 2.0 Array. The gene expression data of all samples were pre-processed through background correction, quantile normalization, probe summarization and probe ID to gene symbol conversion using the MAS5 algorithm in the Affymetrix package (version 1.54.0) of Bioconductor.

Differentially expressed gene (DEG) analysis, data preprocessing and GA-SVM-based classification. The limma package of Bioconductor was used for identification of DEGs. Genes with $\mathrm{P}<0.01$ and a minimum absolute $\log 2(\mathrm{FC})>\log 2(1.5)$ were considered to be differentially expressed between pCR and non-pCR patients. Visualization of the identified DEGs on a heat map was achieved using the ComplexHeatmap version 1.16.0 package (Bioconductor). The GA-SVM method was used as previously described (16) with a search iteration number of 100 and a population size of 20 . Annotations for the probe arrays were obtained using the Affymetrix Human Genome U133A 2.0 Array annotation data (hgu133a2.db version 3.2.3 package; Bioconductor). For cases of multiple probe sets mapping to the same gene, the averages of the probe set values were taken as the expression values.

Functional enrichment analysis of SIRT5. The Pearson's correlation analysis was employed to evaluate statistically significant associations between '221010_s_at' (SIRT5) and other gene probes in the 64 TNBC patients who underwent neoadjuvant doxorubicin/cyclophosphamide therapy followed by paclitaxel treatment in the GSE41998 set. The significance threshold was set at $\mathrm{P}<0.001$ and the resulting correlation networks were visualized with Cytoscape (version 3.5.1; http://cytoscape.org/). Subsequently, to identify the potential underlying mechanisms of SIRT5 in the anthracycline-taxane-based NAC response, we searched genes that were significantly associated with SIRT5 in the Database for Annotation, Visualization and Integrated Discovery (DAVID; version 6.8, david.ncifcrf.gov) (17). In GO analysis, the categories screened were 'cellular component', 'biological process' and 'molecular function' terms. $\mathrm{P}<0.05$ was considered to indicate statistically significant differences.

Oncomine database analysis. The expression levels of SIRT5 in breast cancers were analyzed in Oncomine gene expression array datasets (www.oncomine.org) (18). We searched SIRT5 in the database, using thresholds of $\mathrm{P}<0.05$ and fold change $>1.5$. A total of 6 independent datasets were extracted for the differential expression analysis of SIRT5, through which normal breast tissues were compared with breast cancer tissues. In addition, SIRT5 expression levels were evaluated in breast cancers with differences in clinopathological characteristics, including histological, molecular and pathological subtype and clinical outcome. Additionally, to assess the predictive value of SIRT5 on the response of TNBC patients to neoadjuvant anthracycline-taxane-based chemotherapy, the expression levels of SIRT5 were also examined in epirubicin/cyclophosphamide-docetaxel chemotherapy responders and non-responders.

Survival analysis. The prognostic value of SIRT5 was determined by Kaplan-Meier analysis using the KM plotter online software (http://kmplot.com/analysis/) on 5,143 breast cancer patients (2017 version). SIRT5 gene (probe set, 221010_s_at) was entered into the database (http://kmplot.com/breast/) to obtain Kaplan-Meier survival plot where the number at risk was indicated below the main plot (17). Hazard ratio (HR) with $95 \%$ confidence intervals (CIs) and log-rank P-value were calculated and displayed on the webpage. The primary endpoint of interest was distant metastasis-free survival (DMFS) and the secondary was overall survival (OS). 
Table I. Five core genes identified by using GA-SVM based classifier to discriminate TNBC patients with pCR after anthracycline-taxane based NAC from those without pCR (GSE41998).

\begin{tabular}{llccc}
\hline Probe ID & Gene symbol & P-value & Adjusted P-value & B-statistic \\
\hline 202881_x_at & NOL7 & 0.004161662 & 0.795911511 & -2.86518995 \\
204660_at & GFER & 0.001336072 & 0.762088046 & -2.35709256 \\
209133_s_at & COMMD4 & 0.007963704 & 0.795911511 & -3.15483672 \\
221010_s_at & SIRT5 & 0.006766523 & 0.795911511 & -3.08223489 \\
221281_at & SRC & 0.007015234 & 0.795911511 & -3.0983295 \\
\hline
\end{tabular}

GA-SVM, genetic algorithm-support vector machine; TNBC, triple-negative breast cancer; pCR, pathological complete response; NAC, neoadjuvant chemotherapy.

Statistical analysis. The differentiated expression analysis and Pearson's correlation test were applied for two-class differential expression analyses (e.g., pCR vs. non-pCR) and multiclass ordinal analyses (e.g., grade I vs. II breast cancer) during the Oncomine database analysis (www.oncomine.org). Unless otherwise indicated, in all analyses $\mathrm{P}<0.05$ was considered to indicate a statistically significant difference.

\section{Results}

Identification of DEGs between $p C R$ and non-pCR patients. The overall procedure of analysis in the GEO database is displayed in Fig. 1. Based on the public microarray dataset GSE41998, we first compared gene expression profiles among patients with pCR and non-pCR, and identified 118 DEG probes, including 99 upregulated and 19 downregulated DEGs (Fig. 2A).

GA-SVM-based pCR classifiers. In order to identify optimal biomarkers for predicting pCR, the GA of the GA-SVM method was used to narrow down the number of genes, using the DEGs as features and their expression levels as feature values. A total of 61 gene probes mostly related to the predictive classification were selected (Fig. 2B). Subsequently, these 61 candidate biomarkers were integrated into risk classifiers with regard to the identification of TNBC patients with $\mathrm{pCR}$. SVM analysis with a 10 -fold cross-validation procedure was performed to evaluate the predictive performance of the SVM-based classifiers. The area under the receiver operating characteristic curve was 0.9762 (Fig. 2C). Five core genes were identified to have better predictive performance regarding pCR to NAC. The five core genes were 202881_x_at, 204660_at, 209133_s_at, 221010_s_at, 221281_at (NOL7, GFER, COMMD4, SIRT5 and SRC, respectively; Table I). To the best of our knowledge, SIRT5 is closely related to cell metabolism and had a significant positive association with pCR to NAC in TNBC patients (Fig. 2D). These data indicated the value of SIRT5 in predicting the pCR of TNBC patients to taxane-anthracycline-based NAC.

Functional enrichment analysis of SIRT5. To investigate the potential altered biological functions of SIRT5 associated with the $\mathrm{PCR}$ of TNBC patients to NAC, we first screened 81 probes that were significantly associated with probe '221010_s_at'

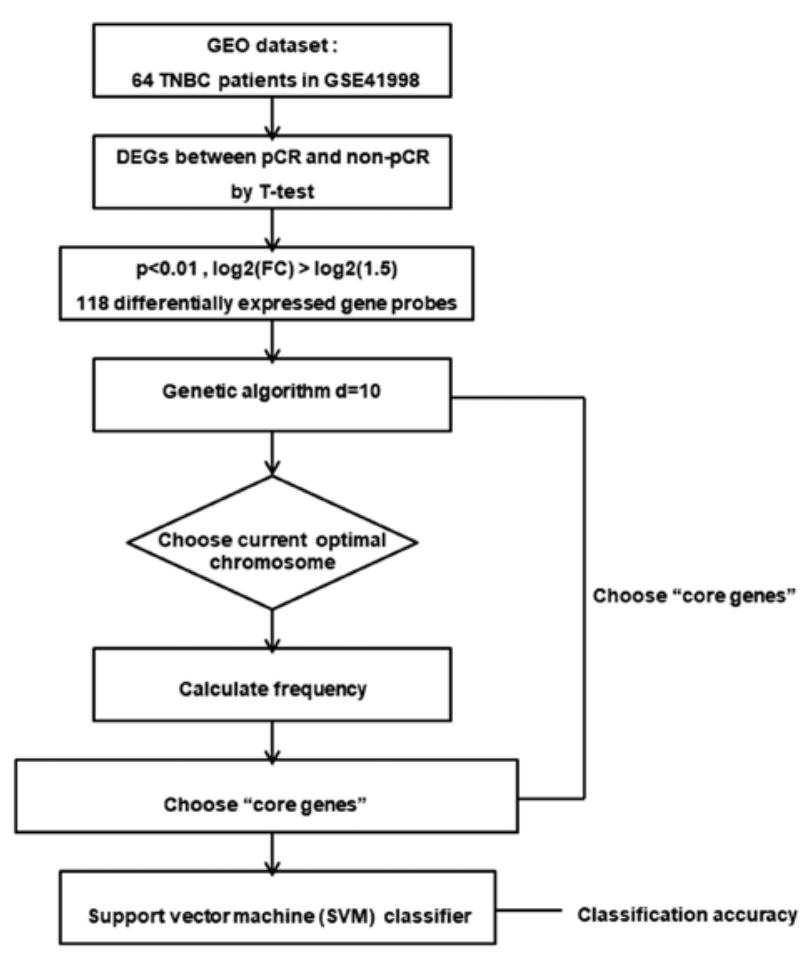

Figure 1. The overall procedure of GEO database analysis. GEO, Gene Expression Omnibus.

(SIRT5) in the TNBC samples in GSE41998 (Fig. 3A). In silico GO analysis of these 81 differentially probes was subsequently performed with a threshold of $\mathrm{P}<0.05$. As displayed in Fig. 3B, SIRT5 was associated with all three GO functions (cellular component, biological process and molecular function), among which the molecular function involved Rho guanyl-nucleotide exchange factor activity ( 3 genes, $\mathrm{P}=0.029)$ and vinculin binding ( 2 genes, $\mathrm{P}=0.034$ ). The cellular component involved extracellular exosomes (20 genes, $\mathrm{P}=0.0047)$, the endoplasmic reticulum membrane (ER) (8 genes, $\mathrm{P}=0.016$ ), hemidesmosomes ( 2 genes, $\mathrm{P}=0.024$ ), the sarcolemma ( 3 genes, $\mathrm{P}=0.028$ ), the plasma membrane ( 20 genes, $\mathrm{P}=0.035$ ), endoplasmic reticulum exit sites ( 2 genes, $\mathrm{P}=0.039$ ) and the basal part of the cell ( 2 genes, $\mathrm{P}=0.039)$. The biological processes involved the IRE1-mediated unfolded protein response (4 genes, $\mathrm{P}=0.001$ ), regulation of Rho protein signal transduction ( 3 genes, $\mathrm{P}=0.03$ ), negative regulation of hormone secretion ( 2 genes, $\mathrm{P}=0.033$ ), 

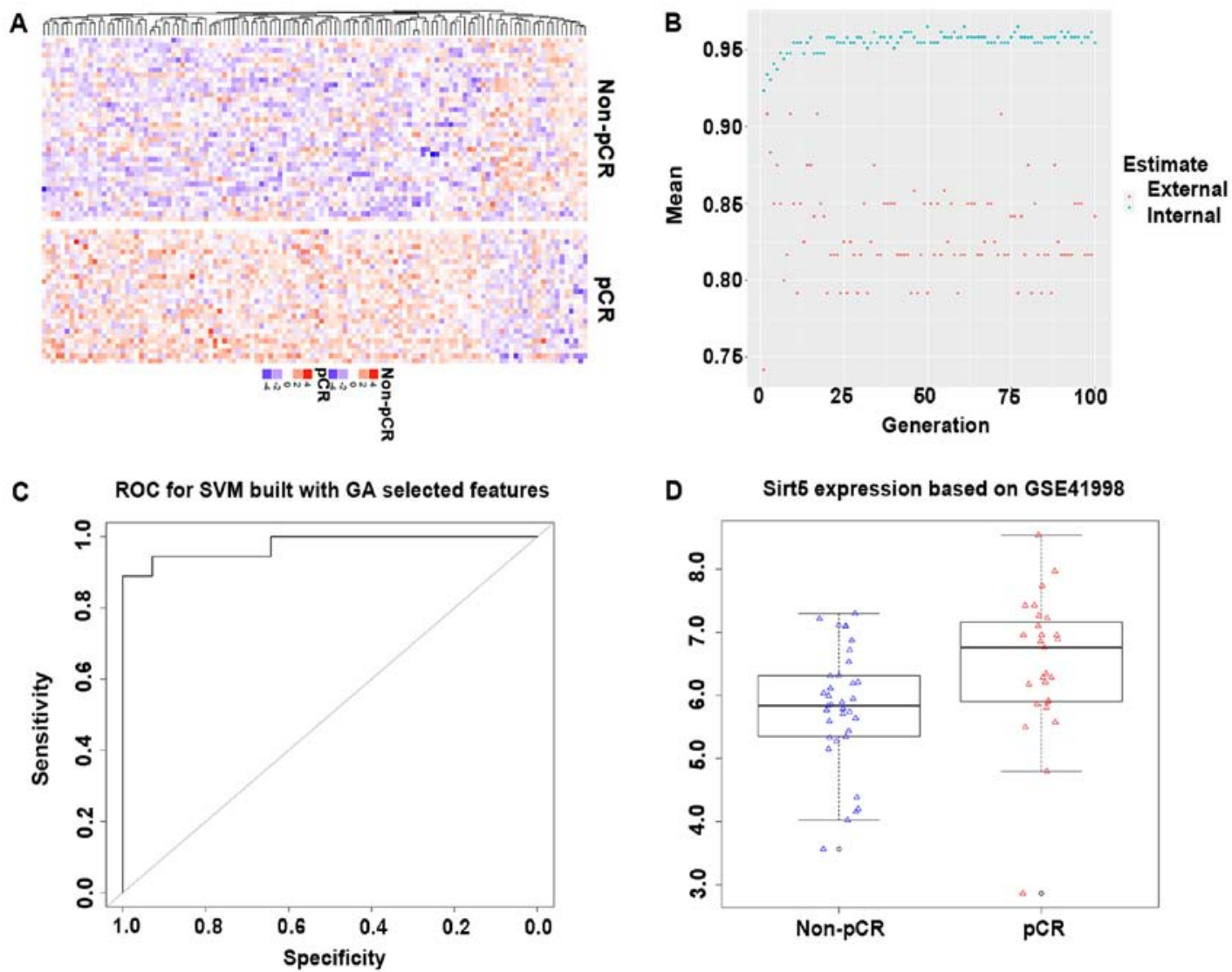

Figure 2. Analysis of 64 TNBC patients with pCR or non-pCR status in GSE41998. (A) The heatmap revealed that 118 probes were significantly differentially expressed between pCR and non-pCR cases. (B) Sixty-one gene probes were screened by GA. (C) The ROC curve for the evaluation of the performance of the GA-SVM-based pCR classifiers. (D) Comparison of SIRT5 expression between patients in the pCR and non-pCR groups. TNBC, triple negative breast cancer; pCR, pathological complete response; GA-SVM, genetic algorithm-support vector machine; SIRT5, sirtuin 5.

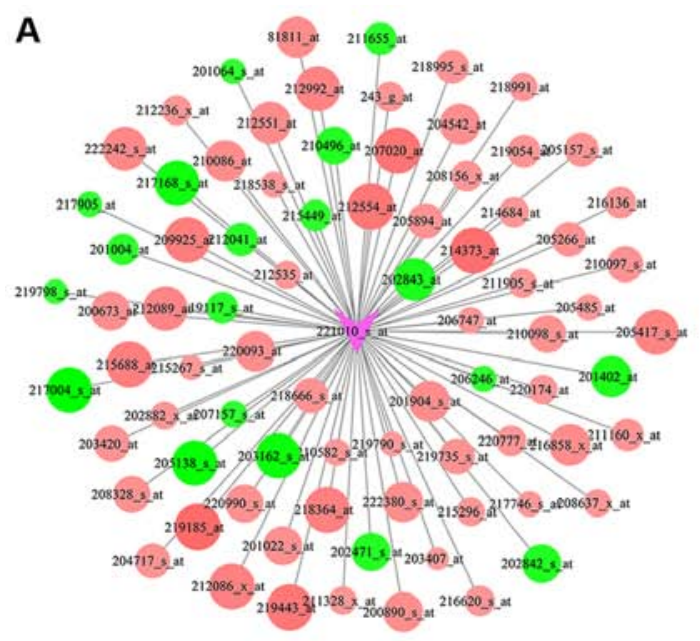

Pearson correlation coefficient

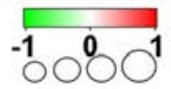

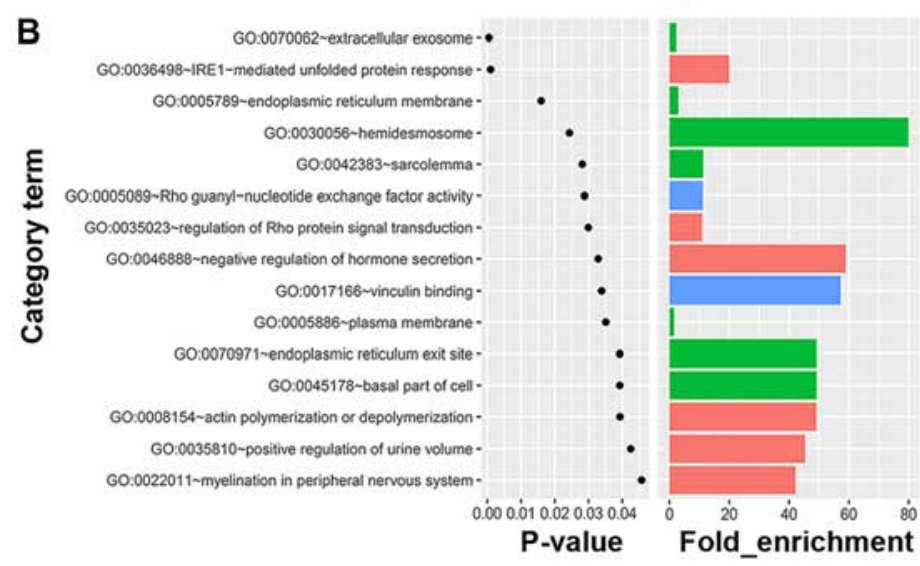

Category

Biological_process

Cellular_component

Molecular_function

Figure 3. Functional enrichment analysis. (A) Eighty-one probes were closely associated with the expression of SIRT5 in the TNBC patients in GSE41998 ( $\mathrm{P}<0.001$ for Pearson's correlation). Red nodes represent positive correlation with '221010_s_at' (SIRT5); green nodes represent negative correlation with '221010_s_at' (SIRT5). (B) Functional enrichment analysis was performed with DAVID. The criterion for selection was P $<0.05$. SIRT5, sirtuin 5; TNBC, triple-negative breast cancer; GO, Gene Ontology.

actin polymerization or depolymerization ( 2 genes, $\mathrm{P}=0.039)$, positive regulation of urine volume ( 2 genes, $\mathrm{P}=0.043)$ and myelination in peripheral nervous system ( 2 genes, $\mathrm{P}=0.046$ ). It is interesting to note that the Rho pathway was reflected in 

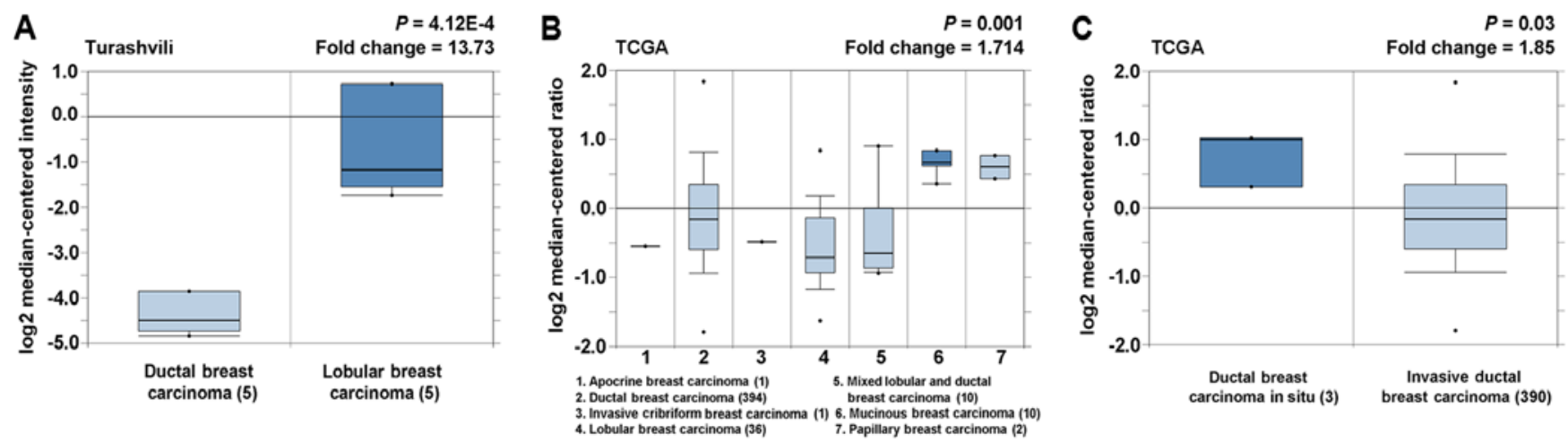

Figure 4. Oncomine database analysis of the expression of SIRT5 in different subtypes of breast cancer. (A) Elevated expression levels of SIRT5 were identified in lobular breast carcinoma (Turashvili breast dataset; Oncomine database), (B) in mucinous breast carcinoma and (C) in ductal breast carcinoma in situ (TCGA breast dataset; Oncomine database). SIRT5, sirtuin 5.
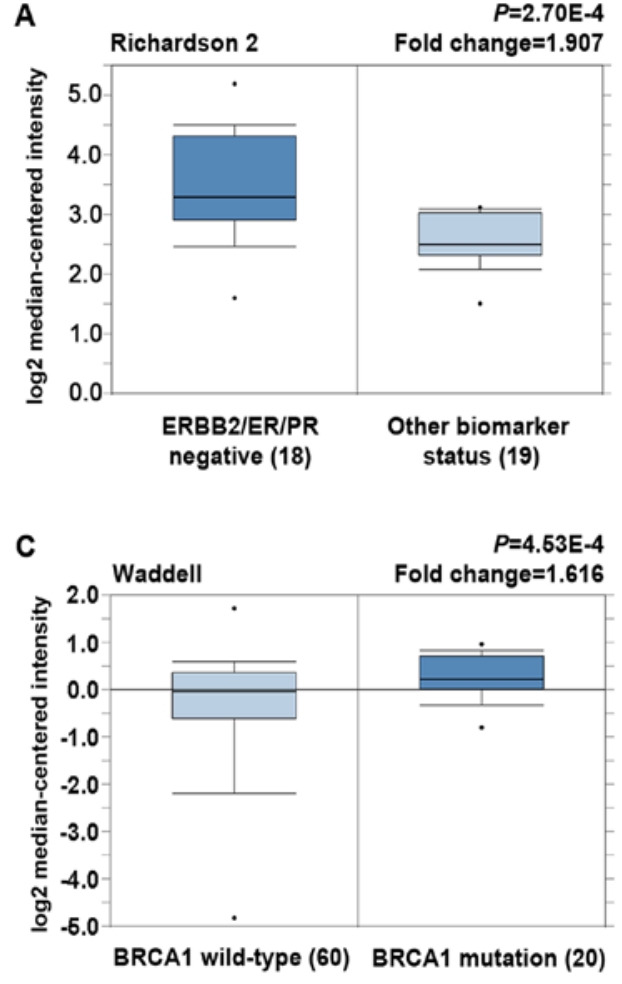

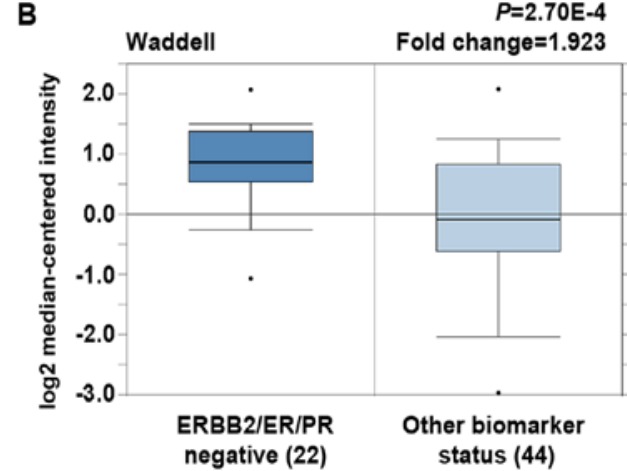

D

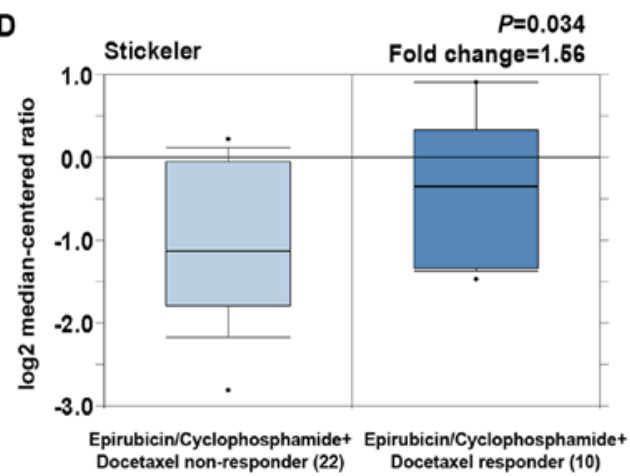

Figure 5. Oncomine database analysis of the expression of SIRT5 in different breast cancer molecular subtypes, BRCA1 mutation and patient treatment responses. (A and B) Elevated expression levels of SIRT5 were identified in TNBC (Richardson breast 2 dataset and Waddell breast dataset; Oncomine database), (C) breast cancer with BRCA1 mutations (Waddell breast dataset; Oncomine database) and (D) andepirubicin/cyclophosphamide-docetaxel responders (Stickeler breast dataset; Oncomine database). SIRT5, sirtuin 5; TNBC, triple-negative breast cancer.

the enriched GO cellular component terms, indicating that aberrations in the Rho pathway may play an important role in the putative SIRT5-mediated response to anthracycline-taxane based NAC.

SIRT5 expression analysis in the Oncomine database. By querying 'SIRT5' in the Oncomine database, the expression of this gene in various cancer types and cancer subtypes was evaluated. In the present study, we further analyzed the expression levels of SIRT5 in breast cancers, through which the log-transformed and normalized expression values of SIRT5 were extracted, analyzed and interpreted from bar charts. On analysis of SIRT5 expression in cancer versus normal tissues, higher expression levels of SIRT5 were observed in breast cancer tissues in three datasets (data not shown), while lower levels were observed in breast cancer tissues in another three datasets (data not shown). These results indicated that SIRT5 may not be a tumorigenic gene in breast cancer.

The results of cancer histological subtype analysis are displayed in Fig. 4. Compared with ductal breast carcinoma, lobular breast carcinoma was found to express higher levels of SIRT5 (Fig. 4A). Overexpression of SIRT5 was observed in mucinous breast carcinoma, relative to the other histological subtypes (Fig. 4B). Meanwhile, ductal breast carcinoma in situ expressed higher levels of SIRT5 than invasive breast 
Table II. Elevated SIRT5 expression in hormone receptor negative or HER2-negative breast cancer tissues compared to hormone receptor positive or HER2-positive breast cancer tissues (Oncomine database).

\begin{tabular}{lcccccc}
\hline Study name & \multicolumn{1}{c}{ Cancer type } & Negative (n) & Positive (n) & Total (n) & Fold change & P-value \\
\hline Waddell breast & ER-negative vs. ER-positive & 27 & 42 & 69 & -1.625 & $9.64 \mathrm{E}-4$ \\
Richardson breast 2 & ER-negative vs. ER-positive & 24 & 15 & 39 & -1.632 & $7.72 \mathrm{E}-4$ \\
Waddell breast & PR-negative vs. PR-positive & 28 & 38 & 66 & -1.570 & 0.003 \\
Richardson breast 2 & PR-negative vs. PR-positive & 26 & 13 & 39 & -1.626 & $5.34 \mathrm{E}-4$ \\
Waddell breast & HER2-negative vs. HER2-positive & 43 & 12 & 55 & -1.816 & 0.013 \\
Richardson breast 2 & HER2-negative vs. HER2-positive & 29 & 8 & 37 & -1.501 & 0.016 \\
\hline
\end{tabular}

SIRT5, sirtuin 5; HER2, human epidermal growth factor receptor 2.

Table III. SIRT5 expression analysis in primary sites relative to metastasis and in different pathological subtypes of breast cancer (Oncomine database).

\begin{tabular}{lcccc}
\hline Study name & Cancer type & Total (n) & Fold change & P-value \\
\hline Radvanyi breast & Primary site vs. metastasis (33 vs. 4) & 37 & 2.398 & $9.53 \mathrm{E}-6$ \\
Bittner breast 2 & Primary site vs. metastasis (327 vs. 9) & 336 & -1.850 & 0.035 \\
Ma breast 4 & Grade 1 vs. grade 2 (4 vs. 3) & 7 & 1.534 & 0.014 \\
Desmadt breast 2 & Grade 2 vs. grade 3 (5 vs. 4) & 9 & -2.127 & 0.028 \\
Bittner breast & Grade 1 vs. grade 2 (5 vs. 23) & 28 & -1.522 & 0.034 \\
Miyake breast 2 & Stage II vs. grade III (97 vs. 18) & 115 & 1.701 & $3.52 \mathrm{E}-5$ \\
Bittner breast & M0 vs. M1 (176 vs. 5) & 181 & -1.523 & 0.026 \\
\hline
\end{tabular}

SIRT5, sirtuin 5 .

carcinoma (Fig. 4C). These results indicated that varying degrees of SIRT5 upregulation may be specific to certain histological subtypes.

With regard to the expression of SIRT5 in different molecular subtypes, SIRT5 was overexpressed in TNBC (Fig. 5A and B). Higher levels of SIRT5 were also expressed in ER-negative, PR-negative and HER2-negative breast carcinoma, compared with ER-positive, PR-positive and HER2-positive carcinomas, respectively (Table II). Furthermore, patients with BRCA1 mutations exhibited elevated SIRT5 levels compared with those expressing wild-type BRCA1 (Fig. 5C). On analysis of SIRT5 expression with regard to patient treatment response, a significant 1.560-fold elevation in SIRT5 expression level was observed in epirubicin/cyclophosphamide-docetaxel responders relative to non-responders (Fig. 5D). Collectively, these results demonstrated that high-level expression of SIRT5 was positively associated with the TNBC and BRCA1 mutant subtypes and the response to anthracycline-taxane based treatment.

On clinical outcome analysis, higher SIRT5 expression was prone to occur in patients presenting with metastasis at 1 and 5 years (Fig. 6A and B). Additionally, in comparison with patients who were still alive at 1 year, those who died within 1 year exhibited SIRT5 overexpression (Fig. 6C). Meanwhile, differential expression of SIRT5 was observed between the primary tumor site and metastatic site, as well as between breast carcinomas with other variable clinicopathological characteristics (grade, stage and distant metastatic status; Table III). Collectively, these results indicated that breast patients with elevated SIRT5 mRNA levels have relatively low 1- and 5-year distant metastasis-free survival rates and 1-year survival rates.

Survival analysis of SIRT5 in breast cancer patients. Subsequently, to evaluate the prognostic effect of SIRT5, we constructed Kaplan-Meier plots and log-rank analyses in an online database. We performed this analysis using the 'best cutoff' value of mRNA of SIRT5 (all percentiles are computed and the best performing threshold is automatically chosen as the cut-off). We observed that high mRNA expression of SIRT5 was an indicator of increased risk for distant metastasis $(\mathrm{P}=0.033$; Fig. 6D). Although it was suggested that SIRT5 may not be a predictor of OS (Fig. 6E), there was predictive value of SIRT5 for early death in breast cancer patients, therefore further study is needed.

\section{Discussion}

NAC is one of the well-established treatments for breast cancer and is particularly relevant in the treatment of TNBCs, as it has been demonstrated that TNBCs have a better response to NAC than other molecular subtypes of breast cancer (19). However, it should be noted that only a minority of patients with TNBC have been reported to achieve a $\mathrm{pCR}$, which has recently 

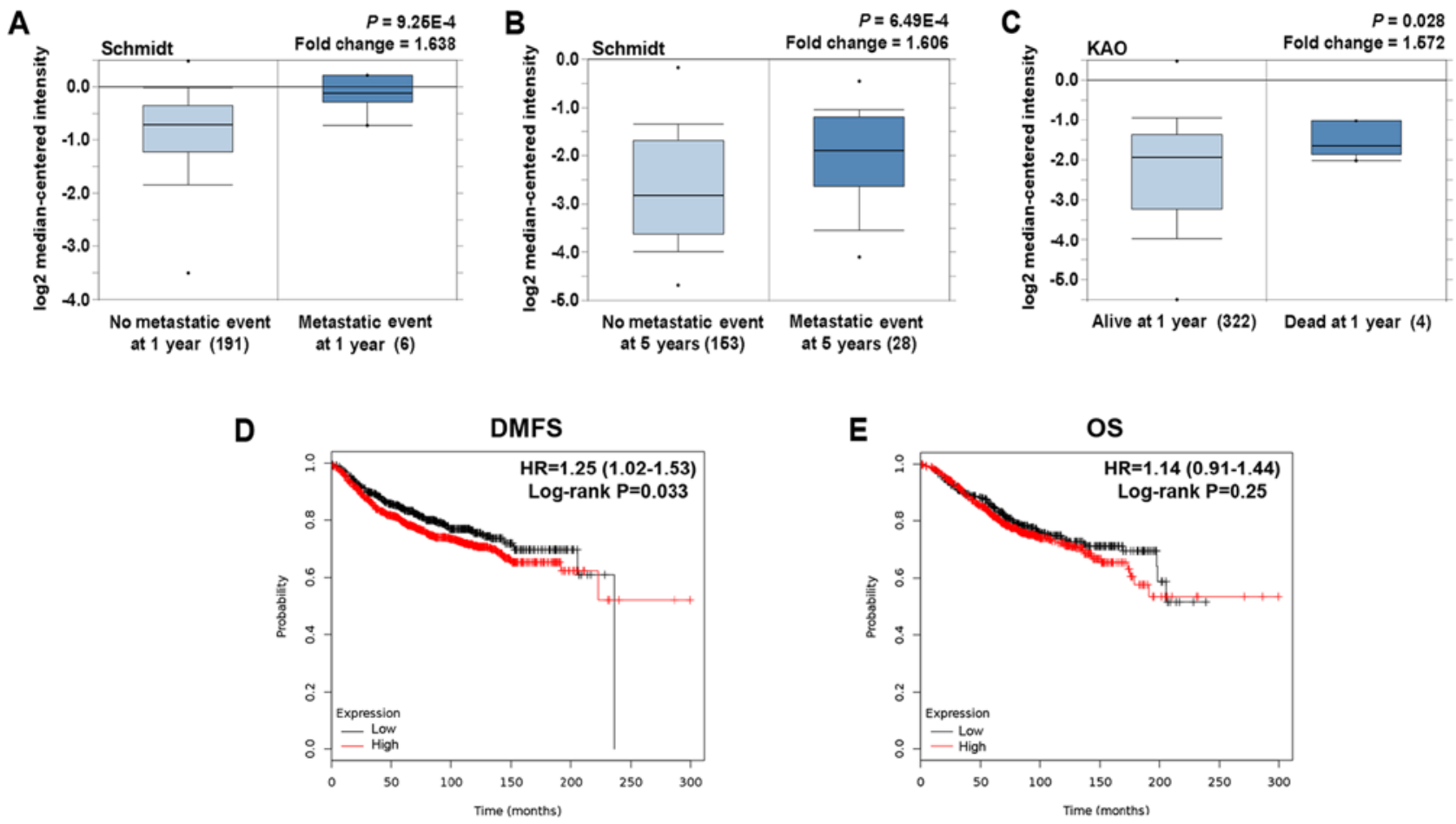

Figure 6. Analysis of the expression of SIRT5 and clinical outcomes of breast cancer patients (Oncomine database and Kaplan-Meier plotter derived from http://kmplot.com/analysis/). Elevated expression levels of SIRT5 were associated with (A) 1-year distant metastasis (Turashvili breast dataset; Oncomine database), (B) 5-year distant metastasis, (C) death at 1 year (TCGA breast dataset; Oncomine database), (D) shorter DMFS and (E) shorter OS. The "best cut-of' for SIRT5 mRNA was used. SIRT5, sirtuin 5; DMFS, distant metastatic-free survival; OS, overall survival.

been proposed as a surrogate for relapse-free survival (RFS) in TNBC patients after NAC and is well-accepted as the strongest predictive factor of good prognosis in patients with TNBC $(20,21)$. Therefore, it is necessary to identify reliable predictors of pCR to NAC in TNBC patients. In the present study, we used high-throughput sequencing data and reported the role of SIRT5 in predicting pCR to anthracycline-taxane-based NAC in TNBC patients for the first time. Using the gene expression and treatment response data of 64 TNBC patients (GSE41998 dataset of the GEO database) who underwent neoadjuvant doxorubicin/cyclophosphamide therapy followed by paclitaxel treatment, SIRT5 was screened as one of the five core genes associated with the pCR of TNBC patients by generating GA-SVM-based pCR classifiers. A positive association was also observed between SIRT5 expression and the pCR to NAC. Additionally, in the Oncomine database analysis, epirubicin/cyclophosphamide-docetaxel responders were found to express elevated levels of SIRT5 when compared with epirubicin/cyclophosphamide-docetaxel non-responders. Collectively, these findings indicated the promising value of SIRT5 in predicting the pCR of TNBC patients to anthracycline-taxane-based NAC.

SIRT5 is one of three mitochondrial SIRTs and has recently been demonstrated to affect cellular metabolism by regulating the post-translational modifications (PTMs) of multiple mitochondrial proteins (22). Previous studies have indicated that SIRT5 can affect ammonia detoxification through the PTMs of carbamoyl phosphate synthetase 1 (23). Results of in vitro experiments in the MDA-MB-231 human breast carcinoma cell line indicated that silenced SIRT5 resulted in the accumulation of ammonia; ammonia-induced autophagy was subsequently activated and enabled tumor cells to survive in stressful conditions, including those induced by chemotherapy (24). Additionally, it was found that autophagy inhibition by proteasome inhibitors increased anthracycline-induced apoptosis in breast cancer cells (25). Thus, it is plausible that elevated SIRT5 expression may increase the sensitivity of cancer cells to anthracycline treatment by promoting ammonia metabolism and inhibiting autophagy.

In addition to cellular metabolism regulation, multiple potential mechanisms through which SIRT5 may affect the response of TNBC patients to NAC were identified by searching genes that were positively correlated with SIRT5 expression in the DAVID database to obtain the GO annotations. First, in the cellular component ontology, the genes were primarily enriched in cell membrane-related structures. The most significant item was extracellular exosomes. Secretion of exosomes containing breast cancer resistance proteins has been suggested to heighten the response of breast cancer cells to doxorubicin (26). In the GO category of molecular function, the enriched items were Rho guanyl-nucleotide exchange factor activity and vinculin binding. Subsequently, it was found that these genes were enriched in a variety of biological processes involved in the endocrine, urinary and nervous systems. Furthermore, it should be noted that the regulation of Rho proteins was also enriched. It has been reported that the Rho signaling pathway exerts its effects on cellular activities primarily through its central role in actin cytoskeleton dynamics (27). A reciprocal relationship has also been explored between Rho proteins and microtubules $(28,29)$ and it has been demonstrated that the activation of the Rho pathway altered the activity of microtubule associated protein 
Tau (MAPT) via phosphorylation to regulate microtubule stabilization (30). MAPT was indicated as a potential predictor of pCR to taxane-containing NAC in advanced breast cancer patients (31). Therefore, these results collectively indicate that SIRT5 may increase the pCR to anthracycline-taxane-based NAC by inhibiting ammonia-induced autophagy and regulating the Rho signaling pathway.

With regard to SIRT5 and tumorigenesis, findings from previous studies have demonstrated that SIRT5 may have different effects on different cancer types by acting as a tumor-suppressor or tumor-promoter. In comparison with normal tissues, SIRT5 was significantly under expressed in head and neck squamous cell carcinoma samples, indicating the role of SIRT5 as a tumor-suppressor (13). Similarly, the expression levels of SIRT5 were substantially downregulated in endometrial carcinoma tissues compared with non-neoplastic endometrium (11). However, no significant change in SIRT5 expression was observed in basal cell carcinoma tissues relative to non-cancerous tissues (12). For breast cancer, a previous study reported significantly elevated expression levels of SIRT5 in breast cancer tissues (14). Nevertheless, in the Oncomine database analysis conducted in the present study, the findings were inconsistent, with either higher or lower expression levels of SIRT5 observed in the breast tumor tissues of different datasets when compared with normal breast tissues. Thus, we speculated that the effects of SIRT5 may even vary in a specific cancer with different clinicopathological characteristics.

To some extent, the results of the present study indeed revealed an association of SIRT5 expression with the clinicopathological characteristics of breast cancer. It was observed that lobular and mucinous breast carcinoma expressed higher levels of SIRT5 in comparison with ductal breast carcinoma. SIRT5 was also overexpressed in breast carcinoma in situ relative to invasive ductal breast carcinoma. These results indicated that SIRT5 upregulation may be specific to certain histopathological type and early-stage breast cancers. However, the reason for this remains unclear at present. Furthermore, compared with other molecular subtypes, the TNBC and BRCA1 mutation subtypes expressed substantially elevated levels of SIRT5. In addition it was observed that elevated expression levels of SIRT5 were significantly associated with 1- and 5-year distant metastasis and with patient death at 1 year. These correlations are consistent with BRCA1 mutations generally being associated with metastatic and aggressive disease, and with TNBC patients having the worst overall and disease-free survival and poor distant metastasis-free survival. Furthermore, the results of online KM plotter analysis also indicated that SIRT5 was a reliable biomarker for DMFS and short OS in breast cancer patients. Collectively, these findings indicated that elevated SIRT5 expression may be involved in the formation of specific histological subtypes of breast cancer and confer a stronger invasive ability on breast cancer cells.

In conclusion, the findings of the present study implicated SIRT5 as a predictor of pCR to anthracycline-taxane-based NAC in TNBC patients. GO analysis indicated that the Rho pathway may be the mechanism through which SIRT5 impacted the response of TNBC to NAC. Additionally, it was found that the expression of SIRT5 was positively correlated with the subtypes of TNBC and BRCA1 mutation, as well as closely associated with poor clinical outcomes and poor prognostic clinicopathological parameters. The results of the present study may provide valuable indications for the basic research and clinical treatment of SIRT5-expressing responders with TNBC to NAC. However, further investigations are required to confirm our hypothesis.

\section{Acknowledgements}

We would like to thank all the participants recruited in the present study.

\section{Funding}

The present study was supported by the National Science and Technology Major Project of the Ministry of Science and Technology of China (no. 2017ZX09304025), the General Project of Liaoning Province Department of Education (no. L2015588), the Science and Technology Plan Project of Liaoning Province (nos. 2016007010, 2015020457 and 2015020458), the Key Research and Development Program of Shenyang (no. 17-230-9-01) and the National Natural Science Foundation of China (no. 81672605).

\section{Availability of data and materials}

The datasets used during the present study are available from the corresponding author upon reasonable request.

\section{Authors' contributions}

LX conceived and designed the study, acquired, analyzed and interpreted the data and wrote the manuscript. YW and XZ analyzed the data. XC, NS, SS, SW, CL, LZ and XQ aided to the critical revision of the manuscript. YT conceived the study, designed the experiments and wrote the manuscript. All authors read and approved the manuscript and agree to be accountable for all aspects of the research in ensuring that the accuracy or integrity of any part of the work are appropriately investigated and resolved.

\section{Ethics approval and consent to participate}

Not applicable.

\section{Consent for publication}

Not applicable.

\section{Competing interests}

The authors declare that they have no competing interests.

\section{References}

1. Yardley DA, Brufsky A, Coleman RE, Conte PF, Cortes J, Glück S, Nabholtz JM, O'Shaughnessy J, Beck RM, Ko A, et al: Phase II/III weekly nab-paclitaxel plus gemcitabine or carboplatin versus gemcitabine/carboplatin as first-line treatment of patients with metastatic triple-negative breast cancer (the tnAcity study): Study protocol for a randomized controlled trial. Trials 17: 63, 2016 
2. von Minckwitz G and Martin M: Neoadjuvant treatments for triple-negative breast cancer (TNBC). Ann Oncol 23 (Suppl 6): vi35-vi39, 2012.

3. Hatzis C, Symmans WF, Zhang Y, Gould RE, Moulder SL, Hunt KK, Abu-Khalaf M, Hofstatter EW, Lannin D, Chagpar AB, et al: Relationship between complete pathologic response to neoadjuvant chemotherapy and survival in triple-negative breast cancer. Clin Cancer Res 22: 26-33, 2016.

4. Song IH, Heo SH, Bang WS, Park HS, Park IA, Kim YA, Park SY, Roh J, Gong G and Lee HJ: Predictive value of tertiary lymphoid structures assessed by high endothelial venule counts in the neoadjuvant setting of triple-negative breast cancer. Cancer Res Treat 49: 399-407, 2017.

5. Vici P, Di Benedetto A,Ercolani C, Pizzuti L, Di Lauro L, Sergi D, Sperati F, Terrenato I, Dattilo R, Botti C, et al: Predictive significance of DNA damage and repair biomarkers in triple-negative breast cancer patients treated with neoadjuvant chemotherapy: An exploratory analysis. Oncotarget 6: 42773-42780, 2015.

6. HumbertO, Riedinger JM, Charon-Barra C, Berriolo-Riedinger A, Desmoulins I, Lorgis V, Kanoun S, Coutant C, Fumoleau P, Cochet $\mathrm{A}$, et al: Identification of biomarkers including ${ }^{18}$ FDG-PET/CT for early prediction of response to neoadjuvant chemotherapy in triple-negative breast cancer. Clin Cancer Res 21: 5460-5468, 2015

7. Yang L, Ma X, He Y, Yuan C, Chen Q, Li G and Chen X: Sirtuin 5: A review of structure, known inhibitors and clues for developing new inhibitors. Sci China Life Sci 60: 249-256, 2017

8. Vellinga TT, Borovski T, de Boer VC, Fatrai S, van Schelven S, Trumpi K, Verheem A, Snoeren N, Emmink BL, Koster J, et al SIRT1/PGC1 $\alpha$-dependent increase in oxidative phosphorylation supports chemotherapy resistance of colon cancer. Clin Cancer Res 21: 2870-9, 2015.

9. Bai L, Lin G, Sun L, Liu Y, Huang X, Cao C, Guo Y and Xie C: Upregulation of SIRT6 predicts poor prognosis and promotes metastasis of non-small cell lung cancer via the ERK1/2/MMP9 pathway. Oncotarget 7: 40377-40386, 2016.

10. Kugel S, Sebastián C, Fitamant J, Ross KN, Saha SK, Jain E, Gladden A, Arora KS, Kato Y, Rivera MN, et al: SIRT6 suppresses pancreatic cancer through control of Lin28b. Cell 165: 1401-1415, 2016.

11. Bartosch C, Monteiro-Reis S, Almeida-Rios D, Vieira R, Castro A, Moutinho M, Rodrigues M, Graça I, Lopes JM and Jerónimo C: Assessing sirtuin expression in endometrial carcinoma and non-neoplastic endometrium. Oncotarget 7: 1144-1154, 2016.

12. Temel M, Koç MN, Ulutaş S and Göğebakan B: The expression levels of the sirtuins in patients with BCC. Tumour Biol 37: 6429-6435, 2016.

13. Lai CC, Lin PM, Lin SF, Hsu CH, Lin HC, Hu ML, Hsu CM and Yang MY: Altered expression of SIRT gene family in head and neck squamous cell carcinoma. Tumour Biol 34: 1847-1854, 2013.

14. Igci M, Kalender ME, Borazan E, Bozgeyik I, Bayraktar R, Bozgeyik E, Camci C and Arslan A: High-throughput screening of Sirtuin family of genes in breast cancer. Gene 586: 123-128, 2016.

15. Horak CE, Pusztai L, Xing G, Trifan OC, Saura C, Tseng LM, Chan S, Welcher R and Liu D: Biomarker analysis of neoadjuvant doxorubicin/cyclophosphamide followed by ixabepilone or Paclitaxel in early-stage breast cancer. Clin Cancer Res 19: 1587-1595, 2013.

16. Peng S, Xu Q, Ling XB, Peng X, Du W and Chen L: Molecular classification of cancer types from microarray data using the combination of genetic algorithms and support vector machines. FEBS Lett 555: 358-362, 2003
17. Györffy B, Lanczky A, Eklund AC, Denkert C, Budczies J, Li Q and Szallasi Z: An online survival analysis tool to rapidly assess the effect of 22,277 genes on breast cancer prognosis using microarray data of 1,809 patients. Breast Cancer Res Treat 123 : 725-731, 2010.

18. Rhodes DR, Kalyana-Sundaram S, Mahavisno V, Varambally R, Yu J, Briggs BB, Barrette TR, Anstet MJ, Kincead-Beal C, Kulkarni P, et al: Oncomine 3.0: Genes, pathways, and networks in a collection of 18,000 cancer gene expression profiles Neoplasia 9: 166-180, 2007.

19. Liedtke C, Mazouni C, Hess KR, André F, Tordai A, Mejia JA, Symmans WF, Gonzalez-Angulo AM, Hennessy B, Green M, et al: Response to neoadjuvant therapy and long-term survival in patients with triple-negative breast cancer. J Clin Oncol 26: 1275-1281, 2008.

20. Caudle AS, Yu TK, Tucker SL, Bedrosian I, Litton JK, Gonzalez-Angulo AM, Hoffman K, Meric-Bernstam F, Hunt KK, Buchholz TA, et al: Local-regional control according to surrogate markers of breast cancer subtypes and response to neoadjuvant chemotherapy in breast cancer patients undergoing breast conserving therapy. Breast Cancer Res 14: R83, 2012.

21. Li J, Chen S, Chen C, Di G, Liu G, Wu J and Shao Z: Pathological complete response as a surrogate for relapse-free survival in patients with triple negative breast cancer after neoadjuvant chemotherapy. Oncotarget 8: 18399-18408, 2017.

22. Kumar S and Lombard DB: Mitochondrial sirtuins and their relationships with metabolic disease and cancer. Antioxid Redox Signal 22: 1060-1077, 2015.

23. Nakagawa T, Lomb DJ, Haigis MC and Guarente L: SIRT5 Deacetylates carbamoyl phosphate synthetase 1 and regulates the urea cycle. Cell 137: 560-570, 2009.

24. Polletta L, Vernucci E, Carnevale I, Arcangeli T, Rotili D, Palmerio S, Steegborn C, Nowak T, Schutkowski M, Pellegrini L, et al: SIRT5 regulation of ammonia-induced autophagy and mitophagy. Autophagy 11: 253-270, 2015.

25. Guo W, Wang Y, Wang Z, Wang YP and Zheng H: Inhibiting autophagy increases epirubicin's cytotoxicity in breast cancer cells. Cancer Sci 107: 1610-1621, 2016.

26. Kong JN, He Q, Wang G, Dasgupta S, Dinkins MB, Zhu G, Kim A, Spassieva $S$ and Bieberich E: Guggulsterone and bexarotene induce secretion of exosome-associated breast cancer resistance protein and reduce doxorubicin resistance in MDA-MB-231 cells. Int J Cancer 137: 1610-1620, 2015.

27. Hanna S and El-Sibai M: Signaling networks of Rho GTPases in cell motility. Cell Signal 25: 1955-1961, 2013.

28. Wojnacki J, Quassollo G, Marzolo MP and Cáceres A: Rho GTPases at the crossroad of signaling networks in mammals: Impact of Rho-GTPases on microtubule organization and dynamics. Small GTPases 5: e28430, 2014.

29. Hervé JC and Bourmeyster N: Rho GTPases at the crossroad of signaling networks in mammals. Small GTPases 6: 43-48, 2015.

30. Amano M, Kaneko T, Maeda A, Nakayama M, Ito M, Yamauchi T, Goto H, Fukata Y, Oshiro N, Shinohara A, et al: Identification of Tau and MAP2 as novel substrates of Rho-kinase and myosin phosphatase. J Neurochem 87: 780-790, 2003.

31. Li ZH, Xiong QY, Tu JH, Gong Y, Qiu W, Zhang HQ, Wei WS, Hou YF and Cui WQ: Tau proteins expressions in advanced breast cancer and its significance in taxane-containing neoadjuvant chemotherapy. Med Oncol 30: 591, 2013. 